\title{
Reconsiderando la clasificación psicopatológica desde el punto de vista psicoanalítico-relacional. Lo histérico/ histriónico como modelo.
}

The psychopathological classification revisited from the psychoanalytic-relational standpoint. The hysterical / histrionic as a model.

\begin{abstract}
Carlos Rodríguez Sutil a , Alejandro Ávila ${ }^{\mathrm{b}}$, Augusto Abello ${ }^{\mathrm{c}}$, Manuel Aburto ${ }^{\mathrm{c}}$, Rosario Castaño ${ }^{\mathrm{c}}$, Susana Espinosa ${ }^{c}$, Ariel Liberman ${ }^{c}$.

${ }^{a}$ Doctor en Psicología, Psicólogo Clínico, Psicoterapeuta de orientación psicoanalítica, Madrid, España. ${ }^{b}$ Catedrático de Personalidad, Evaluación y Tratamiento Psicológicos de la Universidad Complutense. Madrid, España. ${ }^{C}$ Psicólogo Clínico, Psicoterapeuta de orientación psicoanalítica. Miembro Titular del Instituto de Psicoterapia Relacional, España. (Integrantes del Colectivo Grita: Colectivo-Grupo de Estudio e Investigación de la Técnica Analítica, que se ocupa de reconsiderar la Clínica Relacional, fundado en Madrid en 1996 por Alejandro Ávila).
\end{abstract}

Correspondencia: Carlos Rodríguez Sutil (crsutil56@gmail.com)

Recibido: 02/12/2012; aceptado: 01/05/2013

RESUMEN: En el momento presente la clasificación psicopatológica destaca por su gran complejidad y confusión, que da lugar a incontables debates. Realizamos una indagación en este terreno partiendo del concepto de "histeria" por tratarse de un diagnóstico proteico y esquivo, que en cierta medida se solapa con el más de moda, pero no menos confuso, "trastorno límite", y con otros trastornos, como la psicosis, lo que hace de él un caso paradigmático dentro de la discusión diagnóstica en general y psicoanalítica en particular. Su presentación multiforme posiblemente ha llevado, en tiempos de positivismo dominante, a su desprestigio y práctica eliminación de los sistemas de clasificación oficiales. Sin embargo, cuando hablamos de "diagnóstico" al referirnos a la personalidad no debemos entenderlo tanto en el sentido de agrupación de signos y síntomas sino como la valoración y descripción de esas pautas complejas de comportamiento cuyo nivel privilegiado de análisis es el de la relación interpersonal, relación que cuando se constituye como área principal de desadaptación permite hablar de "Trastornos de la personalidad". Concluimos que, a pesar de su complejidad, la personalidad histriónica $(\mathrm{PH})$ es un patrón relacional que conserva una presencia muy importante en la clínica psicoterapéutica, en salud mental y en otros ámbitos de la salud, y que precisamente su adecuada valoración y diferenciación de otros patrones relacionales permitirá afinar los sistemas clasificatorios futuros.

PALABRAS CLAVE: Psicopatología, Clasificación, Trastornos de la Personalidad, Histeria, Trastorno Histriónico de la Personalidad, Trastorno Límite de la Personalidad, Psicoterapia Psicoanalítica.
ABSTRACT: Nowadays psychopathological classification is characterized by a great complexity and confusion, which produces innumerable debates. We make here an inquiry focusing on the concept of "hysteria" as starting point, for it is an elusive and protean diagnostic term, which overlaps to some extent to the more fashionable - but not less fuzzy - one of "borderline disorder", as well as to other disorders, the psychosis for instance, making it a paradigmatic case inside the general discussion in psychopathology and psychoanalysis. In these times of positivist thinking the multiform presentation of hysteria has led to its discredit and virtual elimination from the official classification systems. However, when we use the word "diagnostics" for the personality we should not take it as a mere aggregate of signs and symptoms but as the appraisal and description of those complex behavioral patterns whose privileged level of analysis is the interpersonal relationship. When the relationship is the main area of maladjustment constitutes just what is called "personality disorder". We reach the conclusion that histrionic personality is, in spite of its complexity, a relational pattern with a huge presence in the psychotherapeutic clinic, in mental health and in other domains of the health care system, and that its accurate assessment and differentiation from other patterns will improve future classification systems. KEY WORDS: Psychopathology, Classification, Personality Disorders, Hysteria, Histrionic Personality Disorder, Borderline Personality Disorder, Psychoanalytic Psychotherapy. 
A no ser que Dios le hable a uno directamente acerca de la definición del TLP, es probablemente inútil el aventurarse en esta área en estos momentos. Está claro que Dios le ha hablado a algunos acerca de este asunto, pero, tal como es Su voluntad, lo ha hecho de maneras diferentes y contradictorias [...] No podemos buscar refugio bajo las definiciones del DSM-III y el DSM-III-R. Si algo es seguro, es que esas definiciones no son la obra de Dios, sino la obra de un comité

[Leichtman, 1989. P. 229, traducción de CABrera Abreu, 2002 (48)].

\section{Introducción}

La reciente publicación de un trabajo de Sam Gerson (1) sobre la histeria, en Psychoanalytic Dialogues, con los interesantes comentarios de Muriel Dimen (2) y Richard Lasky (3) ha servido de acicate a nuestro interés por dilucidar la importancia del diagnóstico psicopatológico en psicoterapia psicoanalítica y en salud mental. En nuestro intento ante tarea tan compleja tomamos precisamente el concepto de "histeria" por tratarse de un diagnóstico proteico y huidizo lo que hace de él el paradigma dentro de la problemática diagnóstica en general y psicoanalítica en particular. En este sentido uno de los libros de referencia sobre este asunto, el de Krohn (4), tiene un título significativo: Hysteria, The Elusive Neurosis (Histeria, la neurosis esquiva). En tiempos de positivismo esto posiblemente ha llevado a plantearse su práctica desaparición de los sistemas de clasificación oficiales (5) y, en coherencia con ello, a sugerir la falta de utilidad del término, como ilustran noticias con el título ¿Existe Realmente la Histeria? (El País, 17-10-2006).

Los términos "histeria" y sus derivados - como "neurosis histérica", "histeria de conversión", etc. - han sido retirados progresivamente de los manuales de diagnóstico más relevantes. La palabra "histeria", como es sabido, proviene del griego como un derivado de la palabra útero, puesto que se suponía que este órgano era la causa de la enfermedad histérica. Por otra parte, es de uso común utilizar el término de forma descalificadora y es muy importante que las palabras que aluden a los trastornos mentales no se presten a un mal uso en la medida de lo posible. En los clásicos cuadros histéricos no se encontraba una base orgánica para filiar los trastornos sensorio motores de las pacientes - parecía un trastorno esencialmente femenino - por lo que no podría decirse que hubiera una degeneración del sistema nervioso propiamente dicha.

Como sostiene Daniel Widlöcher (6): "Asistimos, pues, hoy en día, a una diversificación de los enfoques psicopatológicos. No podemos hablar más de un sistema explicativo único, sino de una pluralidad de modelos y métodos". Los manuales de psicopatología al uso distinguen dos tipos de histeria: de conversión y de disociación. Según nuestra experiencia ambas formas son ahora infrecuentes. En cambio la personalidad histérica o histriónica $(\mathrm{PH})$ sigue siendo uno de los "males" de nuestro tiempo. La historia de la histeria es ese complejo entramado 
ORIGINALES Y REVISIONES

de descripciones, adjetivaciones, reacciones, juicios valorativos a los que le viene muy bien esa idea de Nietzsche cuando sostiene que "lo que tiene historia no tiene definición", y que recuerda al orteguiano: "El hombre no tiene naturaleza, tiene historia". Esta historia pone de relieve lo que han señalado muchos psicoanalistas, esto es, la estrecha relación de la histeria con los contextos socio-culturales en los que ha sido pensada. A comienzos del siglo XX el interés por la histeria declinó. Para Judith Herman (7) había sido el impulso político anticlerical de los ilustrados lo que fundamentó el interés científico por la histeria. Una vez establecida una sociedad laica, y siendo la causa de las mujeres una cuestión de los movimientos feministas, no había motivo para continuar las investigaciones en este campo. Lo cierto es que, desaparecido Charcot, sus sustitutos se empeñaron en desposeer a la histeria de una naturaleza psicopatológica propia al carecer de sustrato orgánico verificable. Las histéricas pasaron a ser, según Babinski, meras simuladoras. En otro orden de cosas, para Bleuler, las psicosis histéricas no eran sino cuadros esquizofrénicos en personalidades premórbidas histéricas (8). Según Ross (9), la descripción que hacía Bleuler de la esquizofrenia es en muchos aspectos similar a la moderna descripción del trastorno disociativo de la identidad. Freud siguió su camino apartándose de la etiología traumática y construyendo su edificio conceptual en torno a las pulsiones y a la represión. Janet, por su parte, que siempre defendió una explicación traumática y un proceso de disociación, quedó silenciado hasta muchos años después.

Destaca este rasgo tan frecuente en algunos pacientes en que la seducción -incluyendo la dinámica de identificación con el deseo del otro- fracasa al tener éxito, en una especie de confusión de lengua que si bien aplaca la angustia los sigue dejando ávidos de un encuentro que les permita usar la experiencia al servicio del enriquecimiento personal. No vamos a ocuparnos de la histeria en su sintomatología más florida y espectacular, propia de la neurosis y cuya presencia ha disminuido ostensiblemente desde que se describió el grande ataque hystérique, sino de la supuesta personalidad o carácter histérico, a menudo poco distinguible o aparente. La histeria, en un sentido amplio, parece ser que ha estallado en las clasificaciones actuales, sus fragmentos se han desperdigado por todo el panorama nosográfico, en las diferentes versiones del DSM (10-12). No obstante, nuestra tesis consiste en que ese dar la espalda a un diagnóstico complejo no supone un avance en nuestro conocimiento por mucho que simplifique la labor diagnóstica. Pensamos que las múltiples formas de histeria reflejan una realidad clínica y, más en general, humana que no se disuelve, y menos se resuelve, mirando hacia otro lado. Tampoco pensamos que sea ventajoso desprendernos sin más de los sistemas clasificatorios en psicopatología y en la clínica, por muchos defectos que posean y consecuencias negativas que a menudo provocan. 
ORIGINALES Y REVISIONES

\section{Sobre el diagnóstico de la personalidad}

Siguiendo el modelo médico, el diagnóstico se alcanza examinando los síntomas y signos que presenta el paciente. Esta forma de elaborar la enfermedad como entidad independiente corre el riesgo, ya señalado por Foucault (13), de caer en un "prejuicio de esencia", según el cual la enfermedad es una entidad que se define por los síntomas que la evidencian pero es, en cierta medida, independiente de ellos. Así se habla a veces de la esquizofrenia oculta bajo síntomas obsesivos. Nuestra forma de entender el trastorno mental, en cambio, consiste en hacerlo equivalente a la configuración de síntomas y signos que lo definen, así como su curso fenomenológico, sin aceptar que exista una separación entre la enfermedad y sus expresiones, evitando entrar por el momento en debates etiológicos. Y esto de la misma manera que también rechazamos la concepción esencialista del inconsciente, estructura interior independiente de sus manifestaciones. De haber una estructura por detrás del trastorno psicológico esta es la 'estructura' de la personalidad, idéntica al conjunto de comportamientos de un individuo en su contexto social, con los demás y consigo mismo. La personalidad ofrece sus propias alteraciones relacionales y sirve de sustrato para la aparición de los síntomas, o es dominada por ellos y alcanza su fragmentación casi total en las psicosis, conglomerado de síntomas y signos en el que la personalidad se diluye. Volviendo a Foucault (14), diremos que "la enfermedad atañe a la situación global del individuo en el mundo: en lugar de ser una esencia fisiológica o psicológica es una reacción general del individuo tomado en su totalidad psicológica y fisiológica".

Cuando hablamos del "diagnóstico" de la personalidad no debemos comprenderlo en el sentido de agrupación de signos y síntomas sino como la valoración y descripción de pautas complejas de comportamiento, cuyo nivel privilegiado de análisis es el de la relación interpersonal, y que cuando se constituye como área principal de desadaptación nos lleva a hablar de "trastornos de la personalidad". La personalidad es lo que uno es, más que lo que uno tiene. Siguiendo la definición de Stephen Mitchell (15):

La personalidad no es algo que uno posee, sino algo que uno hace. Uno desarrolla esquemas constantes, pero éstos no reflejan algo "interior", sino más bien modos aprendidos de enfrentar las situaciones; por ello en cierto sentido siempre responden a las mismas situaciones y a la vez son moldeados por éstas.

Asimismo podemos definir la "personalidad" como las formas relativamente estables de pensar, sentir, comportarse y relacionarse con los otros. Algunos de estos procesos son conscientes y otros inconscientes y automáticos. No hay una línea de separación estricta entre un tipo de personalidad y un trastorno de la misma, pues el funcionamiento humano se distribuye en un continuo. Si nuestros modos de pensar, sentir y actuar son desadaptativos y rígidos, conforman el "trastorno" de 
ORIGINALES Y REVISIONES

la personalidad. Todo ser humano está dotado de una personalidad, que es como decir que tiene un estilo propio. La valoración de la personalidad es, por tanto, una cuestión de estilo, y no de signos discretos y enumerables, lo que hace que no sea fácil su delimitación ni la concordancia en el juicio clínico, más aún en el caso de la histeria en la que el peso en la relación es máximo, según demuestra que conceptos como "transferencia" y "contratransferencia" hayan surgido al hilo del trabajo terapéutico con estos "pacientes".

El campo de la salud mental ha restringido de forma progresiva su perspectiva, centrándose cada mez más en las simples agrupaciones de síntomas. Las evidencias actuales, como las que recoge el Psychodynamic Diagnostic Manual (PDM) (16) sugieren que la sobresimplificación de los fenómenos de la salud mental, en servicio de descripciones más consistentes (fiabilidad) y la posibilidad de evaluar los tratamientos desde el punto de vista empírico (validez), ha comprometido el objetivo de una comprensión de la salud mental y de la psicopatología científicamente más adecuada, sin que haya sido posible alcanzar la deseada fiabilidad, especialmente en la práctica clínica. Destaca en este sistema PDM la relevancia que concede a la estructura de la personalidad como principal organizador de la persona y su trastorno en el contexto humano, perspectiva con la que nos identificamos plenamente. Los síntomas o problemas no pueden ser comprendidos, evaluados o tratados en ausencia de la adecuada comprensión de la vida mental de la persona que los padece. Por ejemplo, un estado de ánimo depresivo puede manifestarse de manera radicalmente diferente en una persona que teme las relaciones y evita experimentar y expresar la mayoría de los sentimientos y en otra que está plenamente implicada en todas sus relaciones y emociones vitales. No existe una única y sola presentación clínica de un fenómeno artificialmente aislado, conocido como depresión.

\section{La personalidad histérica o histriónica}

Freud (17), gracias a su famoso "Caso Dora", descubrió el fenómeno de la transferencia pero también provocó una contratransferencia, concepto al que posiblemente nunca llegó a conceder la importancia debida. Ello no impidió que ofreciera un brillante retrato del carácter histérico, destacando la actitud seductora y de la dramatización. Otro de los mecanismos centrales que encontramos en su dinámica es la tendencia al fracaso en el triunfo, como años después lo llamó el creador del psicoanálisis (18). Con él se muestra que el estado anímico del individuo depende de un equilibrio, digamos, "interno", que es influido, pero no determinado, por la situación externa. En otras palabras, el ser humano es un sistema dentro de otros sistemas (familiar, social, etc.). 
Centrándonos en la seducción, encontramos que el histérico, para Masud Kahn (19), discípulo y seguidor de Donald Winnicott, busca una "solución sexual". En ciertas personas se da un "desarrollo sexual precoz" como modo prevalente de enfrentar las fallas ambientales que amenazan al self en desarrollo y que, de este modo, disocian lo que Winnicott denominó "necesidades del yo" que se oponen en su pensamiento a la idea de deseo o de "necesidades del ello". Remedando el lenguaje de la segunda tópica, diremos que el yo necesita reconocimiento y el ello satisfacción física, siendo la solución sexual - que en un sentido más amplio, preferiríamos llamar "solución de la seducción" - la vía para lograr el reconocimiento a costa de la satisfacción física. Como dice Khan (20):

Al final de sus relaciones los histéricos descubren, de la manera más triste e irónica, la verdadera necesidad negada por ellos al principio. Lo que busca el histérico mediante la solución sexual es esencialmente la facilitación de un funcionamiento inadecuado del yo. Esta disociación [...] crea otra situación desastrosa para él. [...] La rendición sexual al objeto conduce a la amenaza de aniquilación.

El rencor del histérico surge porque el nuevo objeto de amor tampoco ha logrado distinguir entre los deseos del ello y las necesidades del yo (21). La confusión que denuncia el rencor es un reflejo de la propia confusión fruto de la disociación. Los deseos pueden ser procesados intrapsíquicamente mientras que las "necesidades del yo" requieren "un apoyo externo real". Aquí es dónde Khan da un paso sumamente relevante: toma el concepto de Winnicott de "tendencia antisocial" (que él conecta con el acting out) que se caracteriza, entre otras cosas, porque exige un respuesta del medio ambiente para vehiculizar la esperanza de una nueva oportunidad, de una experiencia con el otro que en cierto modo cure la disociación, al entrar en contacto con las necesidades del yo, descifrándolas. Para el histérico es en la experiencia sexual - o en la seducción sin más - donde se expresa esta tendencia. El lenguaje del cuerpo se necesita porque el trauma que ha provocado el problema ocurrió antes de que las palabras adquirieran significado, o bien ha desorganizado la capacidad para el pensamiento verbal.

Siguiendo una línea argumentativa semejante, pero innovadora, aparece la teoría de Ute Rupprecht-Schampera (22-23), según la cual, la histeria se produce tras un fallo en la construcción de la relación triádica, por el fracaso de la función parental auxiliar: el padre no supone apoyo suficiente para superar una relación hijo/a-madre excesivamente absorbente, dominada por el miedo, la depresión o el odio. Como variaciones de este tema central nos parece correcto convocar sugerencias como la de Tizón (24), que habla de una función materna abrumada por la angustia que trasmite de forma catastrófica pero aparentando, desde su narcisismo, tener la panacea contra la angustia, trasmitiendo el doble mensaje de la angustia y de que todo está controlado, algo completamente falso. El conato de rebeldía 
ORIGINALES Y REVISIONES

que esta paradoja puede provocar en la persona explica la dinámica apuntada por Gerson (1) de los efectos dañinos provocados por la humillación en la experiencia y articulación de la propia vida afectiva, o por la "falta de reconocimiento", que apuntara Benjamin (25). Ya no parece que la ablación del clítoris sea una salida adecuada al problema (2) pero la humillación destruye la esperanza de ser un buen objeto en la mente del otro y puede llevar regresivamente a la persona al modo de funcionamiento presimbólico. En la mujer una salida de la humillación es el disfraz que se asume la histérica, incorporando el rol que se asume de ella. Parafraseando a Bromberg (26), diremos que el histérico es alguien que recorre la vida pretendiendo ser quien realmente es. Al mismo tiempo, dentro del histérico grave hay un bebé que se siente no querido y busca un "pecho firme" al que agarrarse (24).

Para conseguir la separación, el niño-a utiliza defensivamente las modalidades de la triangulación edípica, con el fin de lograr la triangulación pre-edípica y obtener una pseudo-separación de la madre. Si la niña es capaz de movilizar el interés del padre, sin mayores experiencias traumáticas, sólo se desarrollará una personalidad histérica moderada o benigna. Pero si tras la aproximación sexualizada al padre se produce una gran desilusión o una retraumatización severa, recurrirá a medidas defensivas adicionales: represión, renegación, disociación y retracción infantil. La represión sirve en esos casos para expulsar de la conciencia los recuerdos de acontecimientos eróticos, incluso incestuosos, y los sentimientos asociados de vergüenza, culpa, rabia, etc. La renegación sirve para mantener la imagen idealizada del padre y para que pueda seguir cumpliendo la posible función de ayuda en la separación respecto de la madre. Todos estos mecanismos defensivos permiten el establecimiento de una fantasía autocontenedora que Ruprecht-Schampera (22-23) ha denominado "Mito Pseudo-edípico" - persiste el estado temprano de no-separación de la madre - y que pueden recordar el tipo cuatro de Zetzel (27) o al pseudo-histérico de Kohut (28), con la diferencia de que el primero sugiere que estos pacientes no son analizables y el segundo que sí. En el sujeto varón la solución histérica es más difícil y amenazadora. Para superar la relación original con la madre, altamente conflictiva y sin esperanza, erotiza sus relaciones con ella. Pero en ambos casos, la traumatización viene determinada por la sobreexcitación en el nivel pre-edípico, y no por el rechazo de la aproximación sexual (23).

La "regresión" al modo diádico es un fenómeno típico en los sujetos con estructura límite, según Ruprecht-Schampera (22-23). Ahora bien, si la relación triádica se da desde el origen, no se puede decir propiamente que haya una regresión sino un deterioro. El histérico, en la conversión, utiliza el cuerpo de forma temporal como objeto sustitutorio (del padre auxiliar). El enfermo psicosomático, en cambio, lo utiliza de forma permanente, sustituye al objeto primario. Consideramos que esto, de paso, explicaría que la conversión histérica más avanzada pueda llegar a ser una expresión simbólica del conflicto - el cuerpo "dice” una frase - mientras 
que la enfermedad psicosomática es "opaca", se mueve en el espacio pre-simbólico. La dependencia de la madre deseada y temida es mayor que en otros sujetos, así como es mayor la amenaza y el riesgo. El objeto castrador inicial es la madre. La enfermedad es utilizada muy a menudo por el beneficio que reporta de llamada de atención a la familia, solapándose así con el llamado "síndrome de Briquet".

Personalidad histriónica grave, trastorno límite de la personalidad y psicosis histérica

James Morrison (29), en su resumen del DSM-IV, define al trastorno límite como un trastorno caracterizado por un control de impulsos, autoimagen, estados de ánimo y relaciones interpersonales inestables. Los sujetos que padecen este trastorno intentan desesperadamente evitar el abandono, tienen problemas en la definición de su autoimagen, impulsos autodestructivos, sentimientos crónicos de vacío y aburrimiento así como reacciones de enfado inapropiadas e incontroladas. También experimentan brevemente ideación paranoide o estados disociativos como respuesta al estrés y relaciones que oscilan de forma extrema entre la idealización y la devaluación. Pero también, añadimos nosotros, entre la autoidealización y la autodevaluación.

En la literatura clínica actual se considera que la patología límite es un síndrome clínico bien diferenciado - el trastorno límite de la personalidad (TLP) - la realidad, sin embargo, no es tan simple, pues el concepto de "límite" o "borderline" conlleva importantes problemas de definición. Estos problemas proceden, en parte, de la naturaleza proteica del trastorno - sólo comparable quizá en su variabilidad con la personalidad histérica - como ejemplifica de manera paradigmática su "estable inestabilidad" (30). Lo límite, como era de esperar, transpira una naturaleza de indefinición, confusión, transición a caballo, a modo de charnela, entre modos de funcionamiento y estados dispares. Una muestra de esta indefinición es sin duda la separación de dos formas de TLP en el sistema ICD de la OMS (31): el límite-límite y el impulsivo. Un individuo que padece un brote esquizofrénico en respuesta a una separación puede ser mal diagnosticado como padeciendo un trastorno límite en el que se ha provocado una extrema "depresión por abandono" (32), y viceversa: individuos con una organización límite de la personalidad a menudo son tomados por esquizofrénicos cuando buscan ayuda con ocasión de un importante malestar y una desorganización causados por una separación.

Frente al debate de si lo borderline es una estructura o un modo de funcionamiento, parece acertada la posición de Luigi Cancrini (33) - desde la perspectiva sistémica-familiar- quien se decanta de forma decidida por la segunda opción. Ahora bien, también es posible entender las estructuras como modos de funciona- 
ORIGINALES Y REVISIONES

miento, en la medida que aceptemos la idea de que su frontera es difusa y porosa; se puede pasar con mayor o menor facilidad de una a otra. Dicho de otro modo, los individuos (y los grupos) tienen a su disposición una serie de patrones de comportamiento que pueden variar dependiendo de las circunstancias. Lo "límite" se nos muestra así como una dimensión de gravedad en cualquier trastorno de la personalidad, aunque suponga una estructura neurótica.

El término "límite", cuando es utilizado por clínicos psicoanalíticos para denotar el nivel de severidad, tiene un sentido diferente de cómo se lo usa en el DSM, en el que sólo un tipo de organización límite (la manifestación más histriónica y dramática de este nivel de severidad) recibe el diagnóstico de TLP. Algunas investigaciones (34) han identificado un tipo anaclítico, de paciente límite, afectivamente lábil y fuertemente dependiente, que se aproxima al TLP del DSM-IV así como a ciertas formas graves de trastorno histriónico de la personalidad. Frente a este sitúa un tipo introyectivo, tendente al estado sobre-ideacional, caracterizado por el aislamiento y el retraimiento social, que muy probablemente recibiría los diagnósticos DSM-IV de trastorno de la personalidad paranoide, esquizoide, u obsesivo.

Otto Kernberg (35-37) distingue organizaciones neuróticas de organizaciones límite, dividiendo las segundas, a su vez, en más severas y menos severas, o de nivel bajo y nivel alto. Así diferencia la personalidad histérica, de nivel neurótico, de la PH, de nivel límite alto. Los trastornos histriónicos los pone en relación con lo que en la historia del psicoanálisis se ha denominado: trastorno "infantil", "histeroide", histéricos de tipo 3 y 4 de Zetzel (27) o histeroide disfórico. Aunque, advierte, el clínico encontrará pacientes que presentan niveles intermedios de psicopatología, de modo que los "tipos puros" pueden considerarse extremos de un continuo, con la posibilidad de formas intermedias.

La organización límite de la personalidad tiene en común con la psicosis la difusión de la identidad y la predominancia de operaciones defensivas primitivas, centradas en la escisión, pero se distingue por la presencia de un buen juicio de realidad, que indica la adecuada diferenciación entre el yo y las representaciones de objeto, surgido - siguiendo a Margaret Mahler - en la fase separación-individuación. Kernberg (35-37) incluye ahí los trastornos: límite, esquizoide, esquizotípico, paranoide, hipomaníaco, la hipocondriasis, el trastorno narcisista (junto con el "síndrome narcisista maligno"), y el trastorno antisocial de la personalidad. El trastorno límite presenta, en su opinión, una dinámica similar al esquizoide pero con la expresión de su patología en las interacciones impulsivas en el campo interpersonal, frente a la expresión en la vida de fantasía y la inhibición social del segundo. Destaca en estos pacientes una marcada tendencia a la idealización. Esta tendencia se apoya en la escisión, ya que consiste en la tendencia a verse a sí mismo y a los otros según categorías morales de "totalmente bueno" o "totalmente malo", es decir, de modo irrealmente positivo (como santos, héroes, o salvadores), 
o irrealmente negativo (como odiosos villanos y abusadores), o de ambos modos en un estilo oscilatorio.

Una de las capacidades que definen al ser humano es la de tener en cuenta los estados mentales tanto propios como de los demás a la hora de comprender y predecir la conducta. En psicología evolutiva a esto se lo ha llamado "una teoría de la mente" (38). El comprender y anticipar correctamente las expectativas e ideas de los demás es mucho más importante que apreciar las circunstancias físicas y los aspectos mecánicos de la interacción humana, de los que parecía ocuparse el conductismo radical. Sin embargo, cuando se producen abusos por parte de los padres, en opinión de Peter Fonagy, se debilita la teoría de la mente en el hijo y se favorece la aparición de la patología límite (39-40). Para el hijo deja de ser algo seguro el pensar sobre los deseos, porque esto supone observar los deseos del progenitor de hacerle daño. Así se inhibe la representación secundaria de los hechos mentales representarse las ideas del otro - lo que aporta ciertos beneficios para el individuo, pues le permite, como quien dice, dar un rodeo frente un dolor mental intolerable. El niño busca confortarse en una fusión regresiva con el objeto, con un 'progenitor rescatador', en la fantasía. El abandono de la representación secundaria es, por tanto, una medida defensiva, aunque extrema.

El diagnóstico de "límite" podría no ser más que una dimensión de inestabilidad o severidad, con el significado que confiere Kernberg (35-37) a la "organización límite", de trastorno grave de la personalidad, a caballo entre las neurosis y las psicosis. La personalidad confusa de algunos sistemas, equivalente de la personalidad límite, se caracteriza por una gran desorganización impulsiva y confusión de sentimientos (41-44). Las oscilaciones de carga-descarga, o de fobiacontrafobia, son menos sobresalientes que en otras personalidades del mismo grupo (explosivo-bloqueado y fóbico), así como la tendencia a la huida de la situación temida, porque se producen de manera más inmediata a una situación de conflicto emocional, es decir, ciclos más breves De esta forma se hacen acreedores al rasgo de "impulsividad" que se les atribuye. Fuera de eso, como advierte Caparrós (4142 ), nos encontramos con una total ausencia de rasgos llamativos, con un torpor psicológico general. Las sensaciones de futilidad e irrealidad que Winnicott (45) atribuye al falso self y Fairbairn (46) al paciente esquizoide, es propia también de estos sujetos y, en general con menor intensidad, de las otras dos personalidades del grupo confusional, $\mathrm{y}$, por su escasa iniciativa aparente; se aproximan a la clásica definición de "psicastenia" o "neurastenia" (47). Un trastorno asociado característico son las tendencias incendiarias, la piromanía. Ahora bien, esta sensación de 'futilidad' es una actitud de indolencia que el histérico puede adoptar en su múltiple representación de papeles. Cabrera Abreu (48) dibuja una situación de gran proximidad entre ambos cuadros. Desde posturas en apariencia tan contrapuestas como la psiquiatría biológica y la crítica feminista, se ha proclamado que los casos de 
ORIGINALES Y REVISIONES

trastorno límite de la personalidad "puros" son inexistentes y que la mayoría de las pacientes examinadas podrían ser diagnosticadas de síndrome de Briquet. Es decir, el equivalente moderno de la histeria es el TLP. En conclusión, tal vez la histeria nunca ha desaparecido o se redescubre en forma de TLP.

Cuadro 1.

Comparación entre el trastorno límite de la personalidad (TLP) y la personalidad histriónica (PH)

\begin{tabular}{|c|c|}
\hline TLP & PH \\
\hline $\begin{array}{l}\text { 1. impulsividad } \\
\text { 2. relaciones intensas pero inestables } \\
\text { 3. enfado intenso e inadecuado } \\
\text { 4. trastorno de la identidad } \\
\text { 5. inestabilidad afectiva } \\
\text { 6. esfuerzos frenéticos para evitar el abandono } \\
\text { 7. amenazas de suicidio } \\
\text { 8. sentimientos crónicos de vacío y aburrimiento }\end{array}$ & $\begin{array}{l}\text { 1. busca ser el centro de atención } \\
\text { 2. sexualmente seductor o provocador } \\
\text { 3. expresión emocional superficial y rápidamen- } \\
\text { te cambiante } \\
\text { 4. utiliza permanentemente el aspecto físico para } \\
\text { llamar la atención sobre sí mismo } \\
\text { 5. autodramatización, teatralidad y exagerada } \\
\text { expresión emocional } \\
\text { 6. Sugestionable }\end{array}$ \\
\hline
\end{tabular}

Resumimos en el cuadro los rasgos que habitualmente se atribuyen en los sistemas oficiales al TLP y a la PH. Con letra negrita intentamos subrayar aquellos rasgos que se podrían atribuir a uno y otro cuadro: TLP: [1, 2, 3, 5 y 6]; PH: [1, 3, 5 y 6]. Ahora bien, los trastornos de la identidad [4] y los sentimientos crónicos de vacío y aburrimiento del límite no son imposibles de hallar en sujetos histéricos alterados o "deprimidos", mientras que las amenazas de suicidio o automutilaciones [7] son más exclusivas del TLP en la segunda parte de la frase, porque amenazas de suicidio como forma de manejo ambiental son frecuentes en histéricos graves. Frente a eso, hemos puesto en cursiva los dos rasgos que nos parecen más exclusivos de la PH [2 y 4], con lo que queremos decir que si se nos presenta un paciente con comportamiento límite pero, al mismo tiempo, aparece seductor y provocador y utiliza su aspecto físico para llamar la atención, probablemente se trate de un histérico grave. Finalmente, a nuestro entender el rasgo 8 del TLP - sentimientos crónicos de vacío y aburrimiento -es bastante difícil de valorar y no es infrecuente en el histriónico que se queja del escaso interés de su vida.

Los trastornos de la identidad del TLP son frecuentes en PH graves. Por ese camino llegamos al asunto igualmente complejo de la definición de las psicosis histéricas. Siendo ya difícil la clasificación de los trastornos psicóticos agudos, el concepto de psicosis histérica no está claramente definido, situándose tanto en los trastornos psicóticos agudos como en los trastornos disociativos (49). Ross (9) considera que la mayoría de aquellos casos que se diagnosticaban como "psicosis histérica" eran de naturaleza disociativa, por ello, la respuesta a tratamientos farmacológicos y electroconvulsivos no solía ser buena. Describe tratamientos de 
ORIGINALES Y REVISIONES

los años sesenta con LSD y cannabis, por entonces en experimentación, en una especie de ensañamiento terapéutico iatrogénico, pues tales drogas no hacían sino incrementar la muy probable disociación fundamental de estos pacientes. Plantea la contratransferencia hostil como un factor interviniente en el tratamiento de este tipo de pacientes "resistentes" a las terapéuticas biomédicas, algo que siempre estuvo presente en la relación terapéutica con los histéricos. El DSM-III (11) nos remite a la psicosis reactiva breve que en el DSM-IV (12) se convertirá en trastorno, igualmente breve, pero que puede ser o no reactivo a acontecimientos estresantes graves.

En los años 70 se han retomado las ideas de Janet sobre la psicopatología disociativa (8). Según Janet, una psicosis podía considerarse histérica si se podía establecer su naturaleza disociativa a través de síntomas como la amnesia, la escisión de la personalidad o los estados de conciencia alterados provocados por traumatismos. Spiegel y Fink (50) planteaban que ante un paciente psicótico con delirios, pérdida de asociaciones, ideas de referencia y afecto inapropiado, aparte de los diagnósticos de esquizofrenia, psicosis afectiva o de origen orgánico, cabía plantear el diagnóstico de psicosis histérica. Además de la capacidad de represión y la actitud de belle indiférence como elementos semiológicos, un factor diagnóstico diferencial importante era la capacidad de ser hipnotizado, que en el caso de pacientes histéricos es muy alta (excepto en estados de agitación intensa), al contrario que en los esquizofrénicos. Posteriormente reconocieron de forma explícita la tendencia disociativa asociada a tal condición.

Van der Hart, Nijenhuis y Steele (51), tras revisar la literatura, concluyen que la psicosis histérica es una psicosis disociativa inducida por un trauma, en la que los estados de trance son reviviscencias de las situaciones traumáticas, y que son susceptibles de tratamiento psicoterapéutico. Proponen etiquetar y conceptualizar la psicosis histérica como psicosis disociativa, y considerarla como una forma de trastorno de estrés traumático con disociación estructural, o sea, con partes disociadas de la personalidad como rasgo dominante. Witztum y Van der Hart (8), opinan que en algunos casos el trastorno parece haberse desarrollado porque un episodio traumático reciente también reactivó recuerdos traumáticos existentes, creando una reacción disociativa extrema. El resultado de esta reactivación puede adoptar formas simbólicas, como sería la experiencia subjetiva de haber sido condenado al infierno y ser torturado por demonios.

Tizón (24) se pregunta si los histéricos pueden alucinar, como pensaban antes los psicoanalistas, o se trata más bien de alucinemas, es decir, comunicaciones fantásticas con el propósito de dramatizar o seducir. Nos evoca el ejemplo de un paciente varón, en la mitad de los cuarenta, con anteriores tratamientos de psicoterapia por un periodo de más de diez años. Es trabajador autónomo (pintor) con grandes problemas económicos. Sospecha que varias personas de su familia han 
ORIGINALES Y REVISIONES

llamado a clientes suyos para desprestigiarle y dejarle sin trabajo. Comenta haber tenido alucinaciones complejas de que iba al cielo y hablaba con Dios o con Jesucristo y que era capaz de prever el futuro, con cosas que luego, en su opinión, se han cumplido. Ha perdido recursos económicos (casas de decoración) por presentarse como Dios. Durante una crisis, según él de esquizofrenia, estuvo ingresado una semana en una planta psiquiátrica. En el contacto, en cambio, no produce en absoluto la sensación de extrañeza, distancia, o "falta de sintonía" que caracteriza a los esquizofrénicos y nos queda la duda sobre la realidad de las alucinaciones aunque el paciente parezca sincero.

\section{Dos viñetas clínicas}

Marina o la personalidad histérica moderada con buenas dotes de seducción Marina tiene 30 años y es la mayor de tres hermanos. Atractiva, con un cuerpo muy cuidado, escultural y llamativo. Se viste con gusto exquisito y marcando mucho su figura. Estudió Derecho. Dice de su madre: "Siempre me llevé mal. Es muy diferente a mí. Clava puñales por la espalda, es fría, distante, te da la mano y luego te la quita. Nunca pude contar con ella para nada". Con las hermanas se lleva mal en general. El hermano menor fue siempre considerado "el buen estudiante", el otro siempre fue el guapo de la familia. Marina, en cambio, cree no haber tenido "título" alguno.

Sus recuerdos infantiles están ligados a injusticias que siempre conllevan exclusión. Cuando llama por teléfono para pedir la primera entrevista aclara: "oye yo voy a ir a tu consulta pero desde ya te digo que el problema lo tiene mi novio y no yo". En la primera entrevista repite lo anterior y cuenta que después de diez años de relación, Marcelo, el novio, la ha dejado. Cuando define a la familia de su novio lo hace con pasión por lo "buenísimos" que son todos. "Es una familia ideal".

En las primeras sesiones dice que ahora se da cuenta que fue tratada por Marcelo como si fuese mercancía de supermercado y no como un producto de una tienda distinguida y exclusiva. En cualquier caso, se toma a sí misma como producto de mercado. Está muy angustiada en esta época, llora todos los días, a veces durante horas. En una sesión comenta:

P- Ayer lloré mucho y muy profundamente, pero sabes que no lloraba como una tía de treinta años... lloraba como una niña”.

T- ¿De cuántos años?

P- De ocho.

T- ¿Por qué lloraría esa niña?

P- Porque se perdió.

T- ¿Dónde? 
P- En el Corte Inglés (Marina había descrito previamente su casa como el Corte Inglés: donde hay de todo, pero donde todo es muy impersonal).

En otra ocasión dice: "Sufro más que una mamá a la que se le murió un hijo. Preferiría tener un cáncer a esto".

Durante estos primeros meses de tratamiento recibe propuestas amorosas y/o sexuales de varios hombres y las rechaza todas sistemáticamente. Se muestra sorprendida de que los hombres la miren y tantas veces le propongan salir, ella se desentiende totalmente de la atracción que genera (pasiva y activamente) en ellos. Durante el primer año de tratamiento, a mediados del mes de enero, se va de vacaciones a la playa. Al regreso muestra una foto a su terapeuta, La playa estaba lejísimos y ella con un bikini muy pequeño aparecía en primer plano, pero el supuesto propósito era enseñar al terapeuta lo bonitas que eran las playas donde estuvo.

La paciente escucha todas las intervenciones relacionadas con su familia con interés y suele responder cosas como: "ya lo sé y no quiero hablar más de la pandilla basura -denominación que ha dado a su familia-, ahora lo único que me interesa es Marcelo". El vínculo terapéutico es fluido y cordial en términos generales. En la transferencia adjudica al terapeuta a menudo tremendos poderes de adivinación. Hace infinidad de preguntas, casi todas en relación a motivaciones y cuestiones alrededor de Marcelo, o de la postura que debe adoptar frente a él: ¿qué decir?, ¿cómo decirlo?, ¿cuándo decirlo?, ¿qué no decir? Preguntas que, en general, el terapeuta no responde.

En una sesión, la paciente advierte: "Como a ti te gusta que te cuente las cosas que me pasan contigo y con el tratamiento te contaré algo: resulta que desde hace algún tiempo cada vez que escucho hablar en argentino pongo la oreja... pero no para escuchar el contenido sino para sentir el tono. Hoy, por ejemplo en el bar donde comí había dos hablando en argentino y me quedé escuchando (en realidad escuchaba solo el tono, no se entendía bien lo que decían).... eso me tranquiliza mucho, me ha pasado en días en que estuve mal y me ha tranquilizado un montón, supongo que será algo subconsciente contigo". Hasta ese momento, el origen nacional del terapeuta había sido objeto de comentarios ambiguos y a veces indirectamente descalificadores.

Esta paciente evolucionó clínica y estructuralmente de forma muy positiva a lo largo de cinco años de terapia. Durante ese tiempo retoma la relación con su novio y mejora de manera apreciable su ansiedad y depresión así como la relación con el terapeuta, al principio con descalificaciones indirectas y "regalos" de escaso valor (regalos "de supermercado" por realizar una compra), pero al final se muestra muy agradecida hacia él, reconociendo el valor del vínculo terapéutico, en el que se ha sentido reconocida. 
ORIGINALES Y REVISIONES

Juan Luis o la personalidad histriónica con funcionamiento límite

Juan Luis tiene 25 años cuando acude a la consulta, anteriormente estuvo ocho años con una colega, especialista en psicoanálisis de niños y adolescentes. Toca la guitarra eléctrica y tuvo bastante éxito con un grupo musical hace seis o siete años, pero en la actualidad no actúa y es mantenido prácticamente por la madre. Tuvo dependencia de heroína, parece ser que fumada, y lleva varios años sin consumir. Consume cannabis con regularidad. No conoció a su padre porque les abandonó siendo él muy pequeño. Parece que es rico y vive en Extremadura. A veces fantasea con la idea de ir a visitarle para conocerle, pero no se llega a decidir por temor a sentirse decepcionado. La novia le abandonó y se fue a vivir a las islas, donde trabaja en temas relacionados con el turismo, pero le llama con frecuencia y viene a verle siempre que pasa por Madrid a visitar a su familia. Juan Luis considera que esta es una situación insatisfactoria y le gustaría romper, pero no se siente capaz. Aunque tiene facilidad para atraer a las mujeres se mantiene fiel a Lola, su novia, si bien reconoce que esto es contradictorio. Ese mismo verano se va a vivir a un piso que la madre alquila para él porque dice no soportar a la pareja de la madre, un hombre que en realidad convive con ellos desde que él era pequeño.

Algunas veces que tiene sesión llama la madre pidiendo instrucciones al terapeuta, porque Juan Luis se encierra en la casa y no quiere hablar con nadie. Éste le dice que le comente a Juan Luis que quiere hablar con él, pero que no haga nada más. En esas ocasiones no acude. Tras año y medio de sesiones tiene un intento de suicidio con pastillas y al ingerir la última llama por teléfono a su madre y le dice: "Si me pasa algo, tú no debes sentirte responsable". Se 'sorprende', no obstante, de que a los pocos minutos los bomberos estuvieran derribando la puerta de su casa. A los dos días llama al terapeuta desde el hospital en el que está ingresado y le pide que hable con el psiquiatra del hospital para que le den el alta. El terapeuta le responde que eso no es necesario, que bastará con que él lo solicite y lo más probable es que se la den. A los pocos minutos confirma que le han dado el alta voluntaria. En esa época llama al terapeuta "papá" de vez en cuando. Sigue sin trabajar aunque a veces le ofrecen acompañar a algún cantante de éxito. Considera que eso es una forma de "venderse" y no está dispuesto. Esta es una situación interpersonal típica en estos pacientes, parece que el esfuerzo necesario para su "salvación" es mínimo y consigue que las personas del entorno se desesperen rogándole que lo haga: que acepte el trabajo que le han ofrecido, que complete la asignatura que le falta para terminar la carrera, que responda afirmativamente a la invitación de su pareja. Cuanto más intensamente desea el otro la "solución", más improbable se vuelve.

La terapia parece entrar entonces en un impase. El "entrenamiento" como paciente sólo le está sirviendo para no indagar con profundidad. Es habitual que le receten medicación antipsicótica, sin resultados aparentes, salvo sensación de atontamiento. Pide al terapeuta que le recete algo para suicidarse, aunque sabe que 
éste no es médico. También sabe que aunque pudiera recetárselo no lo haría. Se trabaja el intento de suicidio como una petición de afecto hacia sus figuras relevantes: su novia, su madre, su padre y el terapeuta. El paciente afirma "Es imposible que nadie me ayude", a lo que el terapeuta responde "Cuando te quieren (ayudar) tú no quieres, y quieres cuando no te quieren".

Al año y medio vuelve a consumir heroína fumada, supuestamente la primera vez después de ocho años. La madre y el padrastro deciden acudir a consulta y Juan Luis acepta. Esta sesión sirve un poco para descentrar la calificación de "inmadurez" que hasta entonces recaía sólo en el paciente. La madre y el padrastro llevan viviendo más de veinte años juntos y todavía no están seguros de si forman una familia. Un par de meses después Juan Luis se 'corta' las venas de las muñecas. Muestra las heridas a su terapeuta con una actitud 'mística', éste se siente muy enfadado y apenas logra decir nada. Quizá habría sido un buen momento para que el terapeuta manifestara su enfado.

Al tercer año de tratamiento parece producirse una evolución positiva, está haciendo cursos para ponerse a trabajar, realiza algunos trabajos temporales, sale con alguna chica durante varios meses y parece haber cesado la conflictiva con su madre y su padrastro. Sin embargo, llega a una sesión y anuncia que será la última. La razón es que no soporta más estar viviendo con la madre y ha decidido establecerse con un amigo, no acepta que ésta le siga pagando la terapia y necesita dinero para vivir. De momento piensa vender una colección de sellos bastante valiosos que le dejó su abuelo. El terapeuta le comenta que no es conveniente que abandone la terapia en esta situación y que, provisionalmente, no se le cobrarán las sesiones hasta que encuentre trabajo, Así sigue acudiendo a consulta de manera aparentemente satisfactoria hasta que unos dos meses después llama la madre y aclara que ha vuelto a consumir heroína y cocaína, que está utilizando el dinero de la terapia, para ello porque nunca se ha ido de casa. Poco después ingresa en una comunidad para la desintoxicación y se pierde el contacto.

\section{Conclusión}

Las dos viñetas ilustran dos formas de personalidad histriónica, más moderada y más grave, mostrando la segunda muchas características comunes con el trastorno límite de la personalidad.

El lógico e indispensable interés por la investigación no debe ocupar el primer lugar frente al interés por ayudar a la persona. Es ya un tópico de la clínica el que, cuanto más se conoce a una persona, menos se sabe su diagnóstico. Además, el diagnóstico es algo que el clínico toma siempre como algo provisional, nunca definitivo. 
ORIGINALES Y REVISIONES

Una actitud terapéutica óptima supone la disposición permanente para reevaluar el diagnóstico inicial a partir de nueva información. La práctica diagnóstica que consiste en aplicar meramente una etiqueta a un individuo no ayuda a que el terapeuta transmita una actitud genuinamente terapéutica, una actitud que se considera necesaria para promocionar un resultado óptimo. Aunque la evaluación del momento actual en la relación terapéutica no debe estar fundamentada exclusivamente en el diagnóstico, estamos a favor de ciertos intentos por crear un sistema de clasificación sistemático. Dicho sistema siempre es reflejo de la propia teoría sobre la psicopatología - cuando no de la propia personalidad del clínico - pero proporciona al clínico un importante esquema de referencia en el proceso de recolectar y organizar la información, por y para la intervención, del que no puede prescindir.

Los rasgos de la personalidad, en nuestra opinión, no han de ser inferidos del comportamiento y atribuidos a una instancia interna al individuo, sino que son nuestra manera de tipificar, de entender, el comportamiento de dicho individuo si bien no obligatoriamente en secuencias breves. Cuando hablamos de "diagnóstico" al referirnos a la personalidad no debemos entenderlo tanto en el sentido de agrupación de signos y síntomas sino como la valoración y descripción de pautas complejas de comportamiento, sobre todo en la relación interpersonal. Existen determinados comportamientos que habitualmente van juntos. En el caso que nos ocupa, el de la histeria, creemos haber suministrado los argumentos necesarios para sustentar la conveniencia de mantenerla como entidad nosológica, por muchas dificultades que presente en la práctica. La personalidad histérica, o histriónica, se puede presentar bajo múltiples capas, como neurosis, somatización, trastorno límite o psicosis, entre otros. Si uno de sus objetivos es producir un efecto en el otro, y con frecuencia el tipo de efecto que produzca es secundario, utilizará todas las estrategias relacionales que estén a su alcance, incluyendo, cómo no, aquellas que tienen cabida en los manuales de psicopatología y que algún paciente descubre y aprende tras unos días de estancia en una planta psiquiátrica.

En definitiva, a pesar de su complejidad, la personalidad histriónica es un patrón relacional que conserva una presencia muy importante en la clínica psicoterapéutica, en salud mental y en otros ámbitos de la salud. Su adecuada valoración y diferenciación de otros patrones relacionales permitirá afinar los sistemas clasificatorios futuros. La realidad de la histeria es compleja como compleja es la realidad humana, pero eso no debiera sorprendernos.

\section{BIBLIOGRAFÍA:}

(1) Gerson S. Hysteria and Humiliation. Psychoanal Dialogues 2011; 21: 517-530.

(2) Dimen M. The mystery of hysteria and the crossroads of power: Commentary on paper by Sam Gerson. Psychoanal Dialogues 2011; 21: 531-537. 
(3) Lasky R. Discussion of "Hysteria and Humiliation": Commentary on Paper by Sam Gerson. Psychoanal Dialogues 2011; 21: 538-547.

(4) Krohn A. Hysteria: The Elusive Neurosis. Madison CT: International Universities Press , 1978.

(5) Esbec E.,Echeburúa,E. La Reformulación de los trastornos de la personalidad en el DSM-5. Actas Esp Psiquiatr 2011; 39: 1-11.

(6) Widlöcher, D. Traité de Psychopathologie. París: Presses Universitaires de France, 1994,p. 14.

(7) Herman J. Trauma y recuperación. Madrid: Espasa Calpe, 2004.

(8) Witzum E., Van der Hart O. Psicosis histérica: revisión histórica y evaluación empírica. En: Moskowitz A., Schäfer I., Dorahy M., editors. Psicosis, trauma y disociación. Madrid: Fundación para la investigación y el tratamieto de la esquizofrenia y otras psicosis, 2011; p. 19-35.

(9) Ross C.A. Schizophrenia.Innovations in diagnosis and treatment. New York: The Haworth Press, 2004.

(10) American Psychiatric Association. Diagnostic and Statistical Manual of Mental Disorders. DSM-III.Washington: APA, 1980

(11) American Psychiatric Association. Diagnostic and Statistical Manual of Mental Disorders. DSM-III-R.Washington: APA, 1987.

(12) American Psychiatric Association. Diagnostic and Statistical Manual of Mental Disorders. DSM-IV.Washington: APA, 1994.

(13) Foucault M. Enfermedad Mental y Personalidad. Barcelona: Paidós, 1961, p. 12.

(14) Id.,p. 16.

(15) Mitchell S.A. Conceptos relacionales en psicoanálisis. Una integración. Mexico: Siglo XXI, 1993 , p. 38.

(16) PDM Task Force. Psychodynamic Diagnostic Manual. Silver Spring, MD: Alliance of Psychoanalytic Organizations, 2006, p. 4,

(17) Freud S. Análisis Fragmentario de una Histeria. "Caso Dora". En Obras Completas, vol. I, 3rd ed. Madrid: Biblioteca Nueva, 1973, pp. 933-1002.

(18) Freud, S. Varios tipos de carácter descubiertos en la labor analítica. En Obras Completas, vol. III, 3rd ed. Madrid: Biblioteca Nueva, 1973, pp. 2413-2428.

(19) Khan M. Locura y soledad. Buenos Aires: Lugar Editorial, 1991.

(20) Id.p. 53,

(21) Id.p. 54

(22) Rupprecht-Schampera U. The Concept of 'early triangulation' as a key to a unified model of hysteria. Int J Psychoanal 1995; 76: 457-473.

(23) Rupprecht-Schampera, U. How current are Fairbairn's ideas on hysteria? En: Scharff J.S., Scharff D.E. editors. The Legacy of Fairbairn and Sutherland. Psychotherapeutic Applications. London: Routledge, 2005; pp. 97-111.

(24) Tizón J.L. La histeria como organización o estructura relacional (2): Aplicaciones psicopatológicas y terapéuticas de esta perspectiva. Intersubjetivo 2004; 1: 162-175.

(25) Benjamin J. Like subjects, love objects: Essays on recognition and sexual difference. New Haven, CT: Yale University Press, 1995.

(26) Bromberg PM. Hysteria, dissociation, and cure. En: Standing in the Spaces: Essays on Clinical Process, Trauma and Dissociation. Hillsdale, NJ: Analytic Press, 1998, pp 223-237.

(27) Zetzel E.R. The So Called Good Hysteric. Int J Psychoanal 1968; 49: 256-260. 
ORIGINALES Y REVISIONES

(28) Kohut H. Thoughts on Narcissism and Narcissistic Rage. Psychoanal Study Child 1972; 7 : $360-400$.

(29) Morrison J. The DSM IV Made Easy. Nueva York: Guilford Press, 1995.

(30) Schmideberg M. The borderline patient. En: Arieti S., editor. American Handbook of Psychiatry. vol. 1. New York: Basic Books, 1959, pp. 398-416.

(31) World Health Organisation. The ICD-10 classification of mental and behavioural disorders. Ginebra: W.H.O., 1992.

(32) Masterson J. Psychotherapy of the borderline adult. Nueva York: Brunner/Mazel, 1976.

(33) Cancrini L. Océano Borderline. Viajes por una patología inexplorada. Barcelona: Paidós, 2007.

(34) Blatt S.J., Auerbach, J. S. Differential cognitive disturbances in three types of "borderline" patients. J Pers Disord 1988; 2: 198-211.

(35) Kernberg O. Trastornos Graves de la Personalidad. México: El Manual Moderno, 1987

(36) Kernberg O. La agresión en las perversiones y en los desórdenes de la personalidad. Buenos Aires: Paidós, 1994.

(37) Kernberg O.APsychoanalitic Theory of Personality Disorders.En:Clarkin J.F.,Lenzenweger M.F, editors. Major Theories of Personality Disorders. Nueva York: Guilford Press, 1996, pp. 106-140

(38) Wellman H. M. The Child's Theory of Mind. Cambridge, MA: Bradford/MIT Press, 1990.

(39) Fonagy, P. (1991). Thinking about Thinking: Some Clinical and Theoretical Considerations in the Treatment of a Borderline Patient. Int J Psychoanal 1991; 72: 639-656.

(40) Fonagy, P. y Target, M. (1996). Playing with reality: 1. Theory of mind and the normal development of spychic reality. Int J Psychoanal 1996; 77, 217-233.

(41) Caparrós Sánchez N. La construcción de la personalidad. Las psicopatías. Madrid: Fundamentos, 1981.

(42) Caparrós Sánchez N. Psicopatología Analítico Vincular. I y II. Madrid: Quipú, 1993.

(43) Rodríguez Sutil C. Clasificación y diagnóstico de la personalidad. Clínica y Salud 1995, 6, 161-173.

(44) Rodríguez Sutil C. Psicopatología Psicoanalítica. Un Enfoque Vincular. Madrid: Biblioteca Nueva, 2002.

(45) Winnicott D. W. Ego Distortion in Terms of True and False Self. En: The Maturational Process and the Facilitating Environment: Studies in the Theory of Emotional Development. New York: International Universities Press, 1965, pp. 140-152.

(46) Fairbairn W.R.D. Schizoid Factors in the Personality. En: Psychoanalytic Studies of the Personality. London: Tavistock, 1952, pp.3-27.

(47) Schneider, K. Las Personalidades Psico $\neg$ páticas. Madrid: Morata, 1974.

(48) Cabrera Abreu C. Del síndrome de Briquet (histeria) al Trastorno Límite de la Personalidad: una revisión basada en la evidencia (recogida por Samuel Guze). Interpsiquis, 2002 http://www.psiquiatria. com/articulos/psiq_general_y_otras_areas/historia/4796/.

(49) García-Valdecasas Campelo J., Vispe Astola A., Díaz Mesa E., Fernández Márquez L. Encuadre nosológico de la "psicosis histérica" versus psicosis agudas: revisión a propósito de un caso. Revista de la Asociación Española de Neuropsiquiatría, XXV, 95, julio/septiembre 2005, 9-23

(50) Spiegel D, Fink R. Hysterical psychosis and hypnotizability. Am J Psychiatry 1979;136:777-781.

(51) Van der Hart O., Nijenhuis E., Steele K. El Yo atormentado. Bilbao: Desclee de Brouwer, 2008. 